\title{
SPOROTRICHOSIS: COMPLICATION AND MIMIC OF RHEUMATIC DISEASES
}

Maria Clara Cardoso Gomes Zampirolli1,^, Clarice Garcia Valadares Xavier ${ }^{1}$, Aurivan Essado Dantas ${ }^{1}$, Matheus Fonseca Cardoso $^{1}$, Leandro Augusto Tanure ${ }^{1}$, Flávia Sena Teixeira Santos ${ }^{1}$, Maria Fernanda Brandão de Resende Guimarães ${ }^{1}$, Maria Raquel Costa Pinto ${ }^{1}$

1.Universidade Federal de Minas Gerais, Belo Horizonte (MG), Brazil.

*Corresponding author: mariaclarazamp@gmail.com

\section{BACKGROUND}

Sporotrichosis is an endemic disease caused by fungi of the genus Sporothrix. In Brazil, the number of cases has increased steadily based on two transmission patterns: a sapronotic and a zoonotic route. In immunosuppressed patients, sporotrichosis presents with peculiarities, including poor prognosis, and it can mimic autoimmune diseases.

\section{CASES REPORT}

A 43-year-old woman with rheumatoid arthritis (RA), using methotrexate (MTX) $25 \mathrm{mg} /$ week with an adequate response, presented with ulcerative, erythematous and nodular lesions in right wrist, extending to ipsilateral forearm. Her daughter had similar lesions but less intense, and both of them had contact with a cat. She was submitted to a cutaneous biopsy that evidenced a lymphohistiocytic dermatitis with neutrophilic abscess suggestive of sporotrichosis. She completed treatment with 6 months of itraconazole associated with reduced doses of MTX. During this time, she had an articular flare that resolved completely after finishing the sporotrichosis treatment and returning to high doses of MTX. A 52-year-old woman with overlap syndrome (scleroderma and systemic lupus erythematosus) in end-stage disease presented with nodular, painful and migratory lesions in both arms and prostration. She was diagnosed with invasive sporotrichosis with pulmonary involvement. Amphotericin B was initiated. However, she died after a long hospitalization, due to complications of septic pulmonary infection and partial bowel obstruction secondary to spontaneous pneumoperitoneum. A 46-year-old man, with previous alcoholism and drug addiction, had recurrent polyarthritis and panuveitis. He was diagnosed with RA and ocular involvement, but had inadequate response to corticoid (CS) and MTX. During investigation, Sporothrix was isolated in synovial fluid and in eye biopsy. He was treated with amphotericin B for 60 days evolving with complete joint improvement. He was maintained with CS and itraconazole.

\section{CONCLUSION}

Sporotrichosis' clinical forms are classified into: fixed cutaneous, lymphocutaneous (80\%), disseminated cutaneous and extracutaneous. Disseminated infection is rare (1\%) and it is a consequence of haematogenous dissemination (HD) or conidia inhalation. Eyes are commonly affected and anterior lesions are associated with inoculation. Immunocompromised patients are highrisk group to have HD, greater fungal load, poor prognosis and prolonged therapy. Treatment is based on the disease extension: itraconazole for localized forms and amphotericin for disseminated diseases and critical patients. This case series demonstrates that immunosuppressed patients, even those with previous diagnosis of autoimmune diseases, who fail to respond to therapy or have atypical manifestation, must be investigated further for an alternative diagnosis or concomitant infection. 